InTERnATIONAL ELECTRONiC JoURnAL OF GEOMETRY

Volume 7 No. 2 PP. 47-60 (2014) C)IEJG

\title{
ON A SPECIAL CONFIGURATION OF LINES AND POINTS IN $\mathbb{P}^{N}$
}

\author{
ALBERTO ALZATI AND GIAN MARIO BESANA
}

(Communicated by H. Hilmi HACISALIHOĞLU)

\begin{abstract}
This note concerns some arrangements of lines in $\mathbb{P}^{N}(\mathbb{C})$ and the condition under which there exists a hyperplane intersecting transversely every line of the given arrangement at a unique point.
\end{abstract}

\section{INTRODUCTION.}

In this note we want to address the following combinatorial problem. Let us fix a set $\mathcal{L}$ of $r$ disjoint lines $\left\{L_{1}, L_{2}, \ldots, L_{r}\right\}$ in $\mathbb{P}^{N}(\mathbb{C})$. Let us pick $r$ distinct points $\left\{P_{1}, \ldots, P_{r}\right\}$ such that $P_{i} \in L_{i}$ for $i=1, \ldots, r$. Under which conditions can one find a hyperplane through $P_{1}, \ldots, P_{r}$ that intersects each line $L_{i}$ exactly at $P_{i}$ ? It easy to see that there are situations in which no such hyperplane exists. For instance, let $\langle.$. denote the linear span of a given subset, and assume that $\operatorname{dim}\left(\left\langle L_{1}, L_{2}, L_{3}, L_{4}\right\rangle\right)=$ $3<N$. Then, for any generic 4 -tuple of points $P_{1}, P_{2}, P_{3}, P_{4}$, chosen respectively on $L_{1}, L_{2}, L_{3}, L_{4}$, every hyperplane in $\mathbb{P}^{N}$ that contains all of these points must also contain all of the lines $L_{1}, L_{2}, L_{3}, L_{4}$.

The above example suggests that the dimension of the linear spans of subsets of $\mathcal{L}$ play a significant role, and that with no additional assumptions on such dimensions one cannot hope to find a general solution. However, if we assume that for any subset $\mathcal{L}^{\prime} \subseteq \mathcal{L}$ the dimension of the corresponding linear span depends only upon the cardinality of $\mathcal{L}^{\prime}$, a suitable general result can be achieved. As we shall see, the hypothesis above is satisfied, for instance, when $\mathcal{L}$ is any subset of $r \leq N$ fibres of a rational scroll embedded in $\mathbb{P}^{N}(\mathbb{C})$.

In Section 2 the main theorem is presented. Section 3 contains a few corollaries showing that, in the situation under consideration, the set of hyperplanes in $\mathbb{P}^{N *}$ (the dual space) satisfying the main hypothesis above with respect to $\mathcal{L}$ is large enough. Section 4 studies the special case in which all lines $L_{i}$ are contained in a rational ruled surface. Finally Section 5 is devoted to an application of the main theorem which was indeed the original motivation for us to address this problem.

Date: Received: June 06, 2013 and Accepted: April 09, 2014.

2010 Mathematics Subject Classification. Primary 14N20, 14J26.

Key words and phrases. arrangements of lines, rational ruled surfaces.

This work is within the framework of the national research project "Geometry on Algebraic Varieties" Cofin 2010 of MIUR. 


\section{The MAIN THEOREM}

Let us consider a set $\mathcal{L}$ as mentioned in $\S 1$. Let us fix a line $L:=L_{1} \in \mathcal{L}$. Let us pick a second line $L_{2}$ such that $\operatorname{dim}\left(\left\langle L_{1}, L_{2}\right\rangle\right)=3$. Then, let us pick a third line $L_{3}$, if it exists, such that $\operatorname{dim}\left(\left\langle L_{1}, L_{2}, L_{3}\right\rangle\right)=5$, and so on. We can proceed in this way, say, only for $h \geq 1$ steps to get $L_{1}, L_{2}, \ldots, L_{h+1}$ with $\operatorname{dim}\left(\left\langle L_{1}, L_{2}, \ldots, L_{h+1}\right\rangle\right)=2 h+1 \leq N$. Now we pick another line $L_{h+2}$, if it exists, such that $L_{h+2}$ intersects $\left\langle L_{1}, L_{2}, \ldots, L_{h+1}\right\rangle$ at one point only; then we pick another line $L_{h+3}$, if it exists, intersecting $\left\langle L_{1}, L_{2}, \ldots, L_{h+1}, L_{h+2}\right\rangle$ at one point only, and so on. If possible, we can proceed in this way, say, only for another $q \geq 1$ steps to get $L_{1}, L_{2}, \ldots, L_{h+1}, L_{h+2}, \ldots ., L_{h+q+1}$ with $\operatorname{dim}\left(\left\langle L_{1}, L_{2}, \ldots, L_{h+1}, L_{h+2}, \ldots ., L_{h+q+1}\right\rangle\right)=$ $2 h+q+1=N$. Then, independently of the number of the remaining lines, if any, $\operatorname{dim}\left(\left\langle L_{1}, L_{2}, \ldots, L_{h+1}, L_{h+2}, \ldots ., L_{h+q+1}, \ldots, L_{p}\right\rangle\right)=N$ for any $h+q+2 \leq p \leq r$.

Notice that the function $d:[1, r] \subseteq \mathbb{N} \rightarrow \mathbb{N}$ such that $d(n)=\operatorname{dim}\left(\left\langle L_{1}, L_{2}, \ldots, L_{n}\right\rangle\right)$ depends upon the order in which our lines were chosen. Here we want to consider only sets $\mathcal{L}$ of $r$ lines in $\mathbb{P}^{N}, r \leq N$, such that $d$ does not depend upon the order. In this case we can prove the following theorem, where $k:=h+q$.

Theorem 2.1. Let $(h, k)$ be a given pair of integers with $1 \leq h \leq k, h+k+1=N$. Let $\mathcal{L}=\left\{L_{1}, \ldots, L_{r}\right\}$, with $2 \leq r \leq N$, be any set of $r$ distinct and disjoint lines in $\mathbb{P}^{N}$, such that, for any subset $\left\{L_{1}, \ldots, L_{\rho}\right\} \subseteq \mathcal{L},(\rho \leq r)$, one has:

1) $\operatorname{dim}\left(\left\langle L_{1}, \ldots, L_{\rho}\right\rangle\right)=2 \rho-1$ when $1 \leq \rho \leq h+1$;

2) $\operatorname{dim}\left(\left\langle L_{1}, \ldots, L_{\rho}\right\rangle\right)=\rho+h$ when $h+2 \leq \rho \leq k+1$;

3) $\operatorname{dim}\left(\left\langle L_{1}, \ldots, L_{\rho}\right\rangle\right)=N$ when $k+2 \leq \rho \leq N$.

Let $W_{r}:=\left\{\left(P_{1}, \ldots, P_{r}\right) \in L_{1} \times \cdots \times L_{r} \simeq\left(\mathbb{P}^{1}\right)^{\times r} \mid \operatorname{dim}\left(\left\langle P_{1}, \ldots, P_{r}\right\rangle\right) \leq r-2\right\}$.

Then $\operatorname{dim}\left(W_{r}\right) \leq r-2$, i.e. $W_{r}$ is a closed subscheme of codimension at least 2 in $\left(\mathbb{P}^{1}\right)^{\times r}:=\mathbb{P}^{1} \times \cdots \times \mathbb{P}^{1}$ ( $r$ times). Moreover, if $2 \leq r \leq h+1$ then $W_{r}$ is empty, if $h+2 \leq r \leq k+1$ then $\operatorname{dim}\left(W_{r}\right) \leq r-h-2$.

Before proving Theorem 2.1 we would like to show that there are concrete situations in which the assumptions of Theorem 2.1 are indeed satisfied.

Lemma 2.1. Let $\mathcal{E}=\mathcal{O}_{\mathbb{P}^{1}}(h) \oplus \mathcal{O}_{\mathbb{P}^{1}}(k)$ with $1 \leq h \leq k, N=h+k+1$, and let $S=\mathbb{P}(\mathcal{E})$, be a smooth, rational, surface embedded as a linear scroll in $\mathbb{P}^{N}(\mathbb{C})$ by its tautological line bundle. Let $L_{1}, L_{2}, \ldots, L_{r}$ be any set of $r$ lines in $\mathbb{P}^{N}$ which are fibres of the scroll $S$, with $2 \leq r \leq N$. Then all the assumptions of Theorem 2.1 hold for $L_{1}, L_{2}, \ldots, L_{r}$.

Proof. Let $T$ be the very ample tautological divisor of $S$. Let $f_{H_{1}}, \ldots, f_{H_{r}}$ be the $r$ fibres of $S$, over the points $H_{1}, \ldots, H_{r}$ of the base curve $C \simeq \mathbb{P}^{1}$, corresponding to $L_{1}, L_{2}, \ldots, L_{r}$. If we consider the linear space of $\mathbb{P}^{N}$ spanned by any subset of $\rho$ lines in $\left\{L_{1}, L_{2}, \ldots, L_{r}\right\}$, corresponding to $\rho$ points in $\left\{H_{1}, \ldots, H_{r}\right\}$, say $H_{1}, \ldots, H_{\rho}$, we have that its dimension is

$$
\begin{aligned}
N-h^{0}\left(S, T-f_{H_{1}} \ldots-f_{H_{\rho}}\right) & =N-h^{0}\left(C, \mathcal{E} \otimes \mathcal{O}_{C}\left(-H_{1} \ldots-H_{\rho}\right)\right) \\
& =N-h^{0}\left(\mathbb{P}^{1}, \mathcal{O}_{\mathbb{P}^{1}}(h-\rho) \oplus\right.
\end{aligned}
$$

Now, if $1 \leq \rho \leq h$ the dimension is $N-(h-\rho+1+k-\rho+1)=2 \rho-1$. If $h<\rho \leq k$ the dimension is $N-(k-\rho+1)=\rho+h$. If $k<\rho$ the dimension is $N$. 
In other words:

$$
\operatorname{dim}\left(\left\langle L_{1}, \ldots, L_{\rho}\right\rangle\right)= \begin{cases}2 \rho-1 & \text { if } 1 \leq \rho \leq h+1 \\ \rho+h & \text { if } h+2 \leq \rho \leq k+1 \\ N & \text { if } k+2 \leq \rho \leq N\end{cases}
$$

Hence assumptions 1), 2), 3) of Theorem 2.1 hold for $L_{1}, L_{2}, \ldots, L_{r}$.

The following remark will be very useful for the proof of Theorem 2.1.

Remark 2.1. Let $\mathcal{L}$ be a set of lines in $\mathbb{P}^{N}$ satisfying the assumptions of Theorem 2.1. Let $\mathcal{L}^{\prime}=\left\{L_{1}, \ldots, L_{r^{\prime}}\right\} \subseteq \mathcal{L}$ be any subset of $\mathcal{L}$, with $2 \leq r^{\prime} \leq r$, having a corresponding subscheme $W_{r^{\prime}}$, defined similarly as in Theorem 2.1. Note that $\mathcal{L}^{\prime}$ satisfies the same assumptions as $\mathcal{L}$, so that to prove Theorem 2.1 one can proceed by induction on $r$ : assuming that $\operatorname{dim}\left(W_{r^{\prime}}\right) \leq r^{\prime}-2$ for any $\mathcal{L}^{\prime} \subseteq \mathcal{L}$ with $r^{\prime} \leq r$, we will show that $\operatorname{dim}\left(W_{r}\right) \leq r-2$.

As suggested by Remark 2.1, the proof of Theorem 2.1 will proceed by induction on $r$, and will make use of a few preliminary Lemmata. The following Lemma collects two simple observations that will facilitate the induction process.

Lemma 2.2. In the assumptions of Theorem 2.1, let $r \geq 3$ and let $m$ be any fixed positive integer. Assume that $\operatorname{dim}\left(W_{r^{\prime}}\right) \leq r^{\prime}-m$ for any subset of $r^{\prime}<r$ lines in $\mathcal{L}$. Then, in order to prove that $\operatorname{dim}\left(W_{r}\right) \leq r-m$, one can assume that for any generic configuration $\left(P_{1}, \ldots, P_{r}\right) \in L_{1} \times \bar{L}_{2} \times \cdots \times L_{r} \simeq\left(\mathbb{P}^{1}\right)^{\times r}$ in $W_{r}$ the following facts are true:

1) $\operatorname{dim}\left(\left\langle P_{1}, \ldots, P_{r}\right\rangle\right)=r-2$

2) $\operatorname{dim}\left(\left\langle P_{1}, \ldots, \widehat{P}_{i}, \ldots, P_{r}\right\rangle\right)=r-2$ for any $i$, where $\widehat{P}_{i}$ is deleted.

Proof. To prove that we can assume 1), let us consider $W_{r}^{\prime}:=\left\{\left(P_{1}, \ldots, P_{r}\right) \in\right.$ $\left.L_{1} \times L_{2} \times \cdots \times L_{r} \simeq\left(\mathbb{P}^{1}\right)^{\times r} \mid \operatorname{dim}\left(\left\langle P_{1}, \ldots, P_{r}\right\rangle\right) \leq r-3\right\} \subseteq W_{r}\left(\right.$ if $\left.r=3 W_{r}^{\prime}=\emptyset\right)$. If we project any $r$-uple of $W_{r}^{\prime}$ onto any product of $r-1$ lines chosen in $\mathcal{L}$ we get a $(r-1)$-tuple of the set $W_{r-1}$ corresponding to those $r-1$ lines. By assumption $\operatorname{dim}\left(W_{r-1}\right) \leq r-1-m$, hence $\operatorname{dim}\left(W_{r}^{\prime}\right) \leq r-1-m+1=r-m$. Therefore if $W_{r}=W_{r}^{\prime}$ then $\operatorname{dim}\left(W_{r}\right) \leq r-m$, so that we can always assume that $W_{r} \supsetneq W_{r}^{\prime}$, i.e. fact 1$)$.

To prove that we can assume 2), choose any $i \in\{1, \ldots, r\}$ and let us consider the closed subscheme $W_{r-1} \subseteq W_{r}$ corresponding to the subset $\left\{L_{1}, \ldots, \widehat{L_{i}}, \ldots, L_{r}\right\} \subsetneq \mathcal{L}$, where $\widehat{L_{i}}$ is removed. Obviously $W_{r-1} \times L_{i} \subseteq W_{r}$. By assumption $\operatorname{dim}\left(W_{r-1}\right) \leq$ $r-1-m$, hence $\operatorname{dim}\left(W_{r-1} \times L_{i}\right) \leq r-1-m+1=r-m$. Therefore if $W_{r}=W_{r-1} \times L_{i}$ then $\operatorname{dim}\left(W_{r}\right) \leq r-m$, so that we can always assume that $W_{r} \supsetneq W_{r-1} \times L_{i}$. As this is true for any $i \in\{1, \ldots, r\}$ we can assume fact 2$)$.

Lemma 2.3. Let $L_{1}, \ldots, L_{h+1}$ be disjoint lines in $\mathbb{P}^{2 h+1}$, with $h \geq 1$, such that their linear span has maximal dimension, i.e. $\left\langle L_{1}, \ldots, L_{h+1}\right\rangle=\mathbb{P}^{2 h+1}$. For any $Q \in$ $\mathbb{P}^{2 h+1}$ let $1 \leq t_{Q} \leq h+1$ be the minimum number of lines among the $L_{i}^{\prime}$ s necessary to have $Q$ contained in their linear span, which has dimension $2 t_{Q}-1$. Let $W_{h+1}(Q):=$ $\left\{\left(P_{1}, \ldots, P_{h+1}\right) \in L_{1} \times L_{2} \times \cdots \times L_{h+1} \simeq\left(\mathbb{P}^{1}\right)^{\times(h+1)} \mid \operatorname{dim}\left(\left\langle Q, P_{1}, \ldots, P_{h+1}\right\rangle\right) \leq h\right\}$. Then $0 \leq \operatorname{dim}\left(W_{h+1}(Q)\right) \leq h+1-t_{Q}$ and if $\operatorname{dim}\left(W_{h+1}(Q)\right)=0$ then $W_{h+1}(Q)$ is a single point. 
Proof. The proof will be conducted in detail for $h=2$. The general case is handled exactly in the same fashion. As the given lines have a linear span of maximal dimension, it is possible to choose a coordinate system in the ambient space $\mathbb{P}^{2 h+1=5}$ such that its $2 h+2=6$ fundamental points belong, pairwise, to the $h+1=3$ given lines. In this situation, let us consider the $(h+2=4,2 h+2=6)$ matrix $M$ whose first $3=h+1$ rows are given by the coordinates of points on the lines $L_{1}, L_{2}, L_{3}$, and where the last row consists of the coordinates of $Q$ :

$$
M=\left[\begin{array}{cccccc}
\alpha_{1} & \beta_{1} & 0 & 0 & 0 & 0 \\
0 & 0 & \alpha_{2} & \beta_{2} & 0 & 0 \\
0 & 0 & 0 & 0 & \alpha_{3} & \beta_{3} \\
x_{0} & x_{1} & x_{2} & x_{3} & x_{4} & x_{5}
\end{array}\right]
$$

For any $Q, W_{3}(Q)$ is given by all possible choices of pairs $\left(\alpha_{i}: \beta_{i}\right) \neq(0,0)$ for which $\operatorname{rk}(M) \leq 3$. It is easy to see that, for any $Q$, there exists at least one such choice of pairs $\left(\alpha_{i}: \beta_{i}\right)$, namely $\left(\alpha_{i}: \beta_{i}\right)=\left(x_{2 i-2}, x_{2 i-1}\right)$ for all pairs $\left.\left(x_{2 i-2}, x_{2 i-1}\right) \neq(0,0)\right)$, hence $\operatorname{dim}\left(W_{3}(Q)\right) \geq 0$.

To get the other side of the stated inequality notice that, as $\left(\alpha_{i}: \beta_{i}\right) \neq(0: 0)$, $M$ can always be transformed into the following matrix

$$
M_{1}=\left[\begin{array}{cccccc}
1 & 0 & 0 & \lambda_{1} & 0 & 0 \\
0 & 1 & 0 & 0 & \lambda_{2} & 0 \\
0 & 0 & 1 & 0 & 0 & \lambda_{3} \\
y_{0} & y_{2} & y_{4} & y_{1} & y_{3} & y_{5}
\end{array}\right]
$$

where $\operatorname{rk}\left(M_{1}\right)=\operatorname{rk}(M), \lambda_{i}=\alpha_{i} / \beta_{i}$ or $\lambda_{i}=\beta_{i} / \alpha_{i}$ respectively when $\beta_{i} \neq 0$ or $\alpha_{i} \neq 0$, and $\left(y_{0}, \ldots, y_{5}\right)$ is a permutation of $\left(x_{0}, \ldots, x_{5}\right) . M_{1}$ can then be further transformed, keeping its rank unaltered:

$$
M_{2}=\left[\begin{array}{cccccc}
1 & 0 & 0 & \lambda_{1} & 0 & 0 \\
0 & 1 & 0 & 0 & \lambda_{2} & 0 \\
0 & 0 & 1 & 0 & 0 & \lambda_{3} \\
0 & 0 & 0 & y_{1}-\lambda_{1} y_{0} & y_{3}-\lambda_{2} y_{2} & y_{5}-\lambda_{3} y_{4}
\end{array}\right]
$$

It is $\operatorname{rk}\left(M_{2}\right) \leq 3$ if and only if:

$$
\begin{cases}\lambda_{1} y_{0} & =y_{1} \\ \lambda_{2} y_{2} & =y_{3} \\ \lambda_{3} y_{4} & =y_{5}\end{cases}
$$

As $\operatorname{dim}\left(W_{3}(Q)\right) \geq 0$, the above system must have at least one solution. If no equation is identically satisfied, then there exists only one solution $\left(\lambda_{1}, \lambda_{2}, \lambda_{3}\right)$, corresponding to a triplet of points, one for each line; in this case $\operatorname{dim}\left(W_{3}(Q)\right)=0$ and the Lemma is proved. If there is only one identically satisfied equation, say $y_{0}=y_{1}=0$, then $\operatorname{dim}\left(W_{3}(Q)\right)=1$ (you can choose an arbitrary point on the first line, but then the other two are determined) and in this case $Q \in\left\langle L_{2}, L_{3}\right\rangle$, hence $t_{Q}=2$ and the Lemma is proved. If exactly two equations are identically satisfied, say $y_{0}=y_{1}=y_{2}=y_{3}=0$, then $\operatorname{dim}\left(W_{3}(Q)\right)=2$ (you can choose arbitrary points on the first two lines, while the last point is uniquely determined), and in this case $Q \in\left\langle L_{3}\right\rangle$ hence $t_{Q}=1$ and the Lemma is proved. As $\left(y_{0}, \ldots, y_{5}\right)$ is a permutation of projective coordinates of $Q$, not all equations can be identically satisfied, so that the Lemma is proved for $h=2$. 
Lemma 2.4. Under the assumptions of Theorem 2.1, further assume that $r \geq h+2$. Let $\mathcal{L}^{\prime}=\left\{L_{1}^{\prime}, L_{2}^{\prime}, \ldots, L_{h+1}^{\prime}\right\}$ be any subset of $h+1$ lines chosen from the given set $\mathcal{L}=\left\{L_{1}, \ldots, L_{r}\right\}$. Let $L \in \mathcal{L} \backslash \mathcal{L}^{\prime}$. Then $L$ intersects the $(2 h+1)$-dimensional linear space $\left\langle L_{1}^{\prime}, L_{2}^{\prime}, \ldots, L_{h+1}^{\prime}\right\rangle$ only at one point $Q$ and such $Q$ does not belong to any linear space spanned by any proper subset of $\mathcal{L}^{\prime}$. Moreover, there exists a unique choice of $P_{i} \in L_{i}^{\prime}$, such that $\operatorname{dim}\left(\left\langle Q, P_{1}, \ldots, P_{h+1}\right\rangle\right) \leq h$.

Proof. Assumption 1) of Theorem 2.1 gives that $\mathcal{L}^{\prime}$ spans a $(2 h+1)$-dimensional linear subspace. Any other line $L \in \mathcal{L} \backslash \mathcal{L}^{\prime}$, cuts this subspace only at one point $Q$, by assumption 2). Moreover, $Q$ can not belong to any linear space spanned by a proper subset of $\mathcal{L}^{\prime}$, otherwise the union of this proper subset and $L$ would contradict assumption 1) of Theorem 2.1. Therefore Lemma 2.3, gives a unique choice of points $P_{i} \in L_{i}^{\prime}$ such that $\operatorname{dim}\left(\left\langle Q, P_{1}, \ldots, P_{h+1}\right\rangle\right) \leq h$.

The above Lemmata will now be combined to provide a proof for Theorem 2.1.

Proof. (of Theorem 2.1).

It is convenient to divide the proof into 4 cases, according to the relative sizes of $r, h$ and $k$.

Case 1: $2 \leq r \leq h+1$. In this case $W_{r}$ is actually empty. To see this, choose a coordinate system in $\mathbb{P}^{N}$ such that $2 r$ points among its $N+1$ fundamental points belong, pairwise, to the $r$ given lines. This is possible by assumption 1). As in the proof of Lemma 2.3, consider the following $(r, N+1)$ matrix whose rows are given by the coordinates of points on each of the given $r$ lines:

$$
\left[\begin{array}{cccccccccc}
\alpha_{1} & \beta_{1} & 0 & 0 & \ldots & 0 & 0 & 0 & \ldots & 0 \\
0 & 0 & \alpha_{2} & \beta_{2} & \ldots & 0 & 0 & \ldots & \ldots & \ldots \\
\ldots & \ldots & \ldots & \ldots & \ldots & \ldots & \ldots & \ldots & \ldots & \ldots \\
0 & 0 & 0 & 0 & \ldots & \alpha_{r} & \beta_{r} & 0 & \ldots & 0
\end{array}\right]
$$

As $\left(\alpha_{i}: \beta_{i}\right) \neq(0: 0)$ for all $i$, it is clear that there always exists a non singular, rank $r$, submatrix and thus $W_{r}=\emptyset$.

Case 2: $2 \leq r \leq k+1$. In this case, induction on $r$ will show that

$$
\operatorname{dim}\left(W_{r}\right) \leq r-h-2 .
$$

This slightly stronger inequality implies the statement and it will be useful in proving the remaining cases. If $k=h$, or $2 \leq r \leq h+1$, there is nothing to prove after Case 1 , so we can assume $h<k$ and $r \geq h+2$ (note that this implies $r \geq 3$ ). Our inductive hypothesis is that the desired inequality (2.1) holds for each subset of $r^{\prime}$ lines contained in $\mathcal{L}$, with $2 \leq r^{\prime}<r$ (recall remark 2.1). Moreover, for a generic $\left(P_{1}, \ldots, P_{r}\right) \in W_{r}$, it is enough to consider the cases that $\operatorname{dim}\left(\left\langle P_{1}, \ldots, P_{r}\right\rangle\right)=r-2$ by part 1) of Lemma 2.2 .

Fix any order for $\mathcal{L}$ and, recalling Lemma 2.4 , let $Q_{1}, Q_{2}, \ldots, Q_{r-h-1}$ be the points of intersection of each of the last $r-h-1$ lines with the linear subspace spanned by the first $h+1$ lines. Let us choose a coordinate system in $\mathbb{P}^{N}$ such that its first $2(h+1)$ fundamental points belong, pairwise, to the first $h+1$ lines, and such that each one of the remaining fundamental points belongs to one of the remaining lines. Notice that these remaining fundamental points are certainly distinct from $Q_{1}, Q_{2}, \ldots, Q_{r-h-1}$. The rows of the following $(r, N+1)$ matrix $M$ are given by the coordinates of the points of the $r$ given lines : 


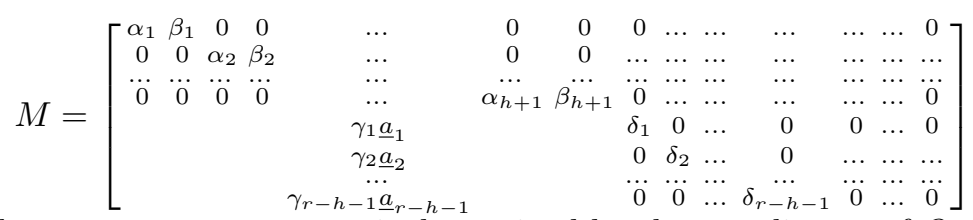

where every row vector $\underline{a}_{i}$ is determined by the coordinates of $Q_{i}$.

By looking at $M$ one sees that an $r$-tuple $\left(P_{1}, \ldots, P_{r}\right)$ belongs to $W_{r}$ (i.e. $\operatorname{rk}(M)<r)$ if and only if $\delta_{i}=0$ for at least one $i$, i.e. if and only if at least a point among $P_{h+2}, \ldots, P_{r}$ coincides with one of the points $Q_{1}, Q_{2}, \ldots, Q_{r-h-1}$. For each of these $r-h-1$ possible equalities one gets a different component of $W_{r}$. Thus it suffices to prove (2.1) for the component with maximal dimension. Without loss of generality, let us assume that the maximal dimension is achieved for $Q_{r-h-1}=P_{r}:=\bar{P}$, the point, on the last line, with coordinates $\left(\gamma_{r-h-1}: \delta_{r-h-1}\right)=(1: 0)$

Let $Z_{r-1}=\left\{\left(P_{1}, \ldots, P_{r-1}\right) \in L_{1} \times L_{2} \times \cdots \times L_{r-1} \mid \operatorname{dim}\left(\left\langle P_{1}, \ldots, P_{r-1}, \bar{P}\right\rangle\right)=r-\right.$ $2\}$. From the above discussion we have that $\operatorname{dim}\left(W_{r}\right) \leq \operatorname{dim} Z_{r-1}$. Note that we can assume $\operatorname{dim}\left(\left\langle P_{1}, \ldots, P_{r-1}, \bar{P}\right\rangle\right)=r-2$ because Lemma 2.2, part 1), guarantees it for a generic point of $W_{r}$, hence it is also true for a generic point of the component of maximal dimension of $W_{r}$. If $Z_{r-1} \subseteq W_{r-1}$ we would have $\operatorname{dim}\left(W_{r}\right) \leq \operatorname{dim}\left(Z_{r-1}\right) \leq$ $\operatorname{dim}\left(W_{r-1}\right) \leq r-1-h-2 \leq r-h-2$, by induction, and we would be done. If not, the generic $(r-1)$-tuple $\left(P_{1}, \ldots, P_{r-1}\right) \in Z_{r-1}$ is such that $\operatorname{dim}\left(\left\langle P_{1}, \ldots, P_{r-1}\right\rangle\right)=r-2$. This fact implies that in any matrix $M$ corresponding to a generic point of $W_{r}$ with $P_{r}=\bar{P}$, it must be $\delta_{r-h-1}=0$, and $\delta_{i} \neq 0$ for any $i \neq r-h-1$. Hence, as $\operatorname{rk}(M)<r$, the submatrix $M_{1}$ consisting of the first $h+1$ rows and the last one must have $\mathrm{rk}\left(M_{1}\right)<h+2$. By Lemma 2.3 , by recalling that $\bar{P}$ can not belong to the linear subspace spanned by any proper subset of $L_{1}, \ldots, L_{h+1}$, this can happen only for a unique choice of points $\left(P_{1}, \ldots, P_{h+1}\right)$. Therefore in any matrix $M$ corresponding to a generic point of $W_{r}$ with $P_{r}=\bar{P}$ all parameters appearing in the first $h+1$ rows and in the last one are fixed. Only $r-(h+1)-1$ parameters remain free in $M$ and we are done.

Case 3: $2 \leq r \leq N$ and $1 \leq r-(h+1) \leq h+1$. In this case, inequality $\operatorname{dim}\left(W_{r}\right) \leq r-2$ will be established by induction on $r$, keeping always in mind Remark 2.1. Having established Cases 1 and 2 we can assume that $k+2 \leq r \leq N$ and, by Lemma 2.2 part 1$)$, we can also assume that $\operatorname{dim}\left(\left\langle P_{1}, \ldots, P_{r}\right\rangle\right)=r-2$ for the generic $r$-tuple of $W_{r}$. Fix an order for $\mathcal{L}$ and let us divide any $r$-tuple in $W_{r}$ into two non empty subsets: $\left(P_{1}, \ldots, P_{r}\right)=\left(P_{1}, \ldots, P_{h+1}\right)\left(P_{h+2}, \ldots, P_{r}\right)$. As $\left(P_{1}, \ldots, P_{r}\right) \in W_{r}$ we have: $\operatorname{dim}\left(\left\langle P_{1}, \ldots, P_{h+1}\right\rangle \cup\left\langle P_{h+2}, \ldots, P_{r}\right\rangle\right) \leq r-2$ and, by Case $1, \operatorname{dim}\left(\left\langle P_{1}, \ldots, P_{h+1}\right\rangle\right)=h$ and $\operatorname{dim}\left(\left\langle P_{h+2}, \ldots, P_{r}\right\rangle\right)=r-(h+1)-1$. Hence $\operatorname{dim}\left(\left\langle P_{1}, \ldots, P_{h+1}\right\rangle \cap\left\langle P_{h+2}, \ldots, P_{r}\right\rangle\right) \geq h+r-(h+1)-1-r+2=0$ and therefore there always exists at least a point $Q \in\left\langle P_{1}, \ldots, P_{h+1}\right\rangle \cap\left\langle P_{h+2}, \ldots, P_{r}\right\rangle$. Moreover, as for the generic $r$-tuple of $W_{r}$ it is true that $\operatorname{dim}\left(\left\langle P_{1}, \ldots, P_{r}\right\rangle\right)=$ $\operatorname{dim}\left(\left\langle P_{1}, \ldots, P_{h+1}\right\rangle \cup\left\langle P_{h+2}, \ldots, P_{r}\right\rangle\right)=r-2$, we can also say that for the generic $r$-tuple of $W_{r}$ there exists a unique point $Q \in\left\langle P_{1}, \ldots, P_{h+1}\right\rangle \cap\left\langle P_{h+2}, \ldots, P_{r}\right\rangle$.

Now, let us consider $\left\langle L_{1}, \ldots, L_{h+1}\right\rangle$ and $\left\langle L_{h+2}, L_{h+3}, \ldots, L_{r}\right\rangle$. As $1 \leq r-(h+$ $1) \leq h+1$ by assumption 1$)$ we can say that $\operatorname{dim}\left(\left\langle L_{1}, \ldots, L_{h+1}\right\rangle\right)=2(h+1)-1$ and $\operatorname{dim}\left(\left\langle L_{h+2}, L_{h+3}, \ldots, L_{r}\right\rangle\right)=2(r-h-1)-1$. As $k+2 \leq r \leq N$ we can say that $\operatorname{dim}\left(\left\langle L_{1}, \ldots, L_{h+1}\right\rangle \cup\left\langle L_{h+2}, L_{h+3}, \ldots, L_{r}\right\rangle\right)=\operatorname{dim}\left(\left\langle L_{1}, \ldots, L_{r}\right\rangle\right)=N$. Hence, if we define $A:=\left\langle L_{1}, \ldots, L_{h+1}\right\rangle \cap\left\langle L_{h+2}, L_{h+3}, \ldots, L_{r}\right\rangle$, we have that $\operatorname{dim}(A)=$ $2 h+1+2 r-2 h-3-N=2 r-2-N \leq r-2$. Moreover, as we saw that 
for the generic $r$-tuple of $W_{r}$ there exists a (unique) point $Q \in\left\langle P_{1}, \ldots, P_{h+1}\right\rangle \cap$ $\left\langle P_{h+2}, \ldots, P_{r}\right\rangle \subseteq A$, we can also say that $A$ is not empty (unless $W_{r}$ is empty, in which case there is nothing to prove). The linear space $A$ contains all intersections points of lines $L_{h+1}, L_{h+2}, \ldots, L_{r}$ with $\left\langle L_{1}, \ldots, L_{h+1}\right\rangle$, and these intersection points surely exist by assumption 2). Therefore $A$ can not be contained in a linear subspace of $\left\langle L_{1}, \ldots, L_{h+1}\right\rangle$ spanned by a proper subset of these lines because no one of those points belong to such a space, thanks to Lemma 2.4. Lemma 2.3 then implies that, for a generic $Q \in A$, there exists a unique $(h+1)$-tuple of points $P_{1}, \ldots, P_{h+1}$, such that $\operatorname{dim}\left(\left\langle Q, P_{1}, \ldots, P_{h+1}\right\rangle\right) \leq h$.

Let us introduce in $L_{1} \times L_{2} \times \cdots \times L_{r} \times A \simeq\left(\mathbb{P}^{1}\right)^{\times r} \times \mathbb{P}^{2 r-2-N}$ the following (non empty) incidence variety:

$$
\begin{aligned}
J:= & \left\{\left(P_{1}, \ldots, P_{r}, Q\right) \in\left(\mathbb{P}^{1}\right)^{\times r} \times A \mid Q \in\left\langle P_{1}, \ldots, P_{h+1}\right\rangle \cap\left\langle P_{h+2}, \ldots, P_{r}\right\rangle\right\} \\
= & \left\{\left(P_{1}, \ldots, P_{r}, Q\right) \in\left(\mathbb{P}^{1}\right)^{\times r} \times A \mid \operatorname{dim}\left(\left\langle Q, P_{1}, \ldots, P_{h+1}\right\rangle\right) \leq h\right. \\
& \text { and } \left.\operatorname{dim}\left(\left\langle Q, P_{h+2}, \ldots, P_{r}\right\rangle\right) \leq r-(h+1)-1\right\} .
\end{aligned}
$$

Let $p: J \rightarrow\left(\mathbb{P}^{1}\right)^{\times r}$ and $f: J \rightarrow A$ be the natural projections. It is $p(J) \subseteq W_{r}$ because if $\left(P_{1}, \ldots, P_{r}, Q\right) \in J$ then the points $\left(P_{1}, \ldots, P_{r}\right)$ can not be linearly independent in $\mathbb{P}^{N}$. On the other hand we have seen that for any $r$-tuple of $W_{r}$ there exist at least a point $Q \in\left\langle P_{1}, \ldots, P_{h+1}\right\rangle \cap\left\langle P_{h+2}, \ldots, P_{r}\right\rangle \subseteq A$ and that for the generic $r$-tuple of $W_{r}$ there exist a unique point $Q$. Hence $\operatorname{Im}(p)=W_{r}$ and $\operatorname{dim}(J)=\operatorname{dim}\left(W_{r}\right)$. Then $\operatorname{dim}\left(W_{r}\right)=\operatorname{dim}(J)=\operatorname{dim}(\operatorname{Im}(f))+\operatorname{dim}($ generic fibre of $f$ ).

Let us consider any point $Q \in A$. As $Q \in\left\langle L_{1}, \ldots, L_{h+1}\right\rangle$, Lemma $2.3 \mathrm{im}$ plies that there exists at least an $(h+1)$-tuple of points $\left(P_{1}, \ldots, P_{h+1}\right)$ such that $\operatorname{dim}\left(\left\langle Q, P_{1}, \ldots, P_{h+1}\right\rangle\right) \leq h$. As $Q \in\left\langle L_{h+2}, L_{h+3}, \ldots, L_{r}\right\rangle$, Lemma 2.3 implies that there exists at least an $(r-h-1)$-tuple of points $\left(P_{h+2}, \ldots, P_{r}\right)$ such that $\operatorname{dim}\left(\left\langle Q, P_{h+2}, \ldots, P_{r}\right\rangle\right) \leq r-h-2$. Therefore $\operatorname{Im}(f)=A$.

In order to estimate the dimension of a generic fiber of $f$, let $Q$ be now a generic point of $A$. Lemma 2.3 implies that $A$ can not be contained in a linear subspace of $\left\langle L_{1}, \ldots, L_{h+1}\right\rangle$ spanned by a proper subset of these lines and that there exists a unique $(h+1)$-tuple of points $\left(P_{1}, \ldots, P_{h+1}\right)$ such that $\operatorname{dim}\left(\left\langle Q, P_{1}, \ldots, P_{h+1}\right\rangle\right) \leq h$. Hence to get a bound for $\operatorname{dim}\left(f^{-1}(Q)\right)$ it suffices to consider the $(r-h-1)$-tuples of points $P_{h+2}, \ldots, P_{r}$ such that $\operatorname{dim}\left(\left\langle Q, P_{h+2}, \ldots, P_{r}\right\rangle\right) \leq r-h-2$. With the notation introduced in the proof of Lemma 2.3, it is true that

$\operatorname{dim}\left(f^{-1}(Q)\right)=\operatorname{dim}\left(W_{r-h-1}(Q)\right)=$

$\operatorname{dim}\left(\left\{\left(P_{h+2}, \ldots, P_{r}\right) \in L_{h+1} \times L_{h+2} \times \cdots \times L_{r} \mid \operatorname{dim}\left(\left\langle Q, P_{h+2}, \ldots, P_{r}\right\rangle\right) \leq r-h-2\right\}\right)$.

If $A$ is not contained in a linear subspace of $\left\langle L_{h+1}, \ldots, L_{r}\right\rangle$ spanned by a proper subset of these lines, Lemma 2.3 gives that for the generic point $Q \in A$ there exists only one $(r-h-1)$-tuple of points $\left(P_{h+2}, \ldots, P_{r}\right)$ such that $\operatorname{dim}\left(\left\langle Q, P_{h+2}, \ldots, P_{r}\right\rangle\right) \leq$ $r-h-2$. In this case $\operatorname{dim}\left(f^{-1}(Q)\right)=0$ and therefore $\operatorname{dim}\left(W_{r}\right)=\operatorname{dim}(J)=$ $\operatorname{dim}(\operatorname{Im}(f))+\operatorname{dim}($ generic fibre of $f)=\operatorname{dim}(A)=2 r-2-N \leq r-2$ and we are done.

If $A$ is contained in at least one linear subspace spanned by a proper subset of $\left\{L_{h+1}, \ldots, L_{r}\right\}$, let $2 t-1$ be the dimension of the space, spanned by $t$ lines, with the minimal dimension among them. Note that $1 \leq t<r-(h+1) \leq h+1$. For all $Q \in A$ Lemma 2.3 gives $\operatorname{dim}\left[W_{r-h-1}(Q)\right] \leq r-h-1-t$. Then we have 
$\operatorname{dim}\left(W_{r}\right)=\operatorname{dim}(J)=\operatorname{dim}(\operatorname{Im}(f))+\operatorname{dim}($ generic fibre of $f) \leq 2 t-1+r-h-1-t=$ $t+r-h-2<h+1+r-h-2=r-1$, i.e. $\operatorname{dim}\left(W_{r}\right) \leq r-2$.

Case 4: $2 \leq r \leq N$ and $h+2 \leq r-(h+1)<k+1$. Because of Cases 1, 2 and 3 we can assume $k+2 \leq r \leq N$ and, by Lemma 2.2 part 1), we can also assume that $\operatorname{dim}\left(\left\langle P_{1}, \ldots, P_{r}\right\rangle\right)=r-2$ for the generic $r$-tuple of $W_{r}$. From Case 2 we have $\operatorname{dim}\left(W_{r-h-1}\right) \leq r-h-1-h-2=r-2 h-3$.

As before, fix an order for $\mathcal{L}$ and let us divide every $r$-tuple in $W_{r}$ into two non empty subsets $\left(P_{1}, \ldots, P_{r}\right)=\left(P_{1}, \ldots, P_{h+1}\right)\left(P_{h+2}, \ldots, P_{r}\right)$. As $\left(P_{1}, \ldots, P_{r}\right) \in W_{r}$ we have that $\operatorname{dim}\left(\left\langle P_{1}, \ldots, P_{h+1}\right\rangle \cup\left\langle P_{h+2}, \ldots, P_{r}\right\rangle\right) \leq r-2$ and, from Case 1, $\operatorname{dim}\left(\left\langle P_{1}, \ldots, P_{h+1}\right\rangle\right)=h$. Moreover, Lemma 2.2 part 2$)$ gives $\operatorname{dim}\left(\left\langle P_{h+2}, \ldots, P_{r}\right\rangle\right)=$ $r-h-2$ for the generic $r$-tuple of $W_{r}$.

Thus $\operatorname{dim}\left(\left\langle P_{1}, \ldots, P_{h+1}\right\rangle \cap\left\langle P_{h+2}, \ldots, P_{r}\right\rangle\right) \geq h+r-h-2-r+2=0$ and therefore there always exists at least one point $Q \in\left\langle P_{1}, \ldots, P_{h+1}\right\rangle \cap\left\langle P_{h+2}, \ldots, P_{r}\right\rangle$. Moreover, as $\operatorname{dim}\left(\left\langle P_{1}, \ldots, P_{r}\right\rangle\right)=\operatorname{dim}\left(\left\langle P_{1}, \ldots, P_{h+1}\right\rangle \cup\left\langle P_{h+2}, \ldots, P_{r}\right\rangle\right)=r-2$, for a generic $r$-tuple of $W_{r}$, it follows that there exists a unique point $Q \in\left\langle P_{1}, \ldots, P_{h+1}\right\rangle \cap$ $\left\langle P_{h+2}, \ldots, P_{r}\right\rangle$.

As in the previous case let us consider $\left\langle L_{1}, \ldots, L_{h+1}\right\rangle$ and $\left\langle L_{h+2}, \ldots, L_{r}\right\rangle$. As $h+2 \leq r-(h+1)<k+1$ by assumptions 1$)$ and 2$)$ we have $\operatorname{dim}\left(\left\langle L_{1}, \ldots, L_{h+1}\right\rangle\right)=$ $2(h+1)-1$ and $\operatorname{dim}\left(\left\langle L_{h+2}, L_{h+3}, \ldots, L_{r}\right\rangle\right)=r-h-1+h=r-1$. As $k+2 \leq r \leq N$ we have $\operatorname{dim}\left(\left\langle L_{1}, \ldots, L_{h+1}\right\rangle \cup\left\langle L_{h+2}, L_{h+3}, \ldots, L_{r}\right\rangle\right)=\operatorname{dim}\left(\left\langle L_{1}, \ldots, L_{r}\right\rangle\right)=N$. As in the previous case, let $A=\left\langle L_{1}, \ldots, L_{h+1}\right\rangle \cap\left\langle L_{h+2}, L_{h+3}, \ldots, L_{r}\right\rangle$. It is $\operatorname{dim}(A)=$ $2 h+1+r-1-N=2 h+r-N$. Moreover, as for the generic $r$-tuple of $W_{r}$ there exists a (unique) point $Q \in\left\langle P_{1}, \ldots, P_{h+1}\right\rangle \cap\left\langle P_{h+2}, \ldots, P_{r}\right\rangle \subseteq A, A$ is non empty, unless $W_{r}$ is empty, in which case there is nothing to prove. Note that $A$ contains all the intersection points of each of the lines $L_{h+2}, \ldots, L_{r}$ with $\left\langle L_{1}, \ldots, L_{h+1}\right\rangle$ and such points certainly exist by assumption 2). Hence $A$ is not contained in any linear subspace of $\left\langle L_{1}, \ldots, L_{h+1}\right\rangle$, spanned by a proper subset of these lines because none of the intersections points mentioned above can be contained in such a subspace by Lemma 2.4. Lemma 2.3 then gives, for a generic point $Q \in A$, a unique $(h+1)$-tuple of points $P_{1}, \ldots, P_{h+1}$, such that $\operatorname{dim}\left(\left\langle Q, P_{1}, \ldots, P_{h+1}\right\rangle\right) \leq h$.

As in the previous case, let us introduce in $L_{1} \times L_{2} \times \cdots \times L_{r} \times A \simeq\left(\mathbb{P}^{1}\right)^{\times r} \times$ $\mathbb{P}^{2 h+r-N}$ the following (non empty) incidence variety:

$$
\begin{aligned}
J:=\{ & \left.\left(P_{1}, \ldots, P_{r}, Q\right) \in\left(\mathbb{P}^{1}\right)^{\times r} \times A \mid Q \in\left\langle P_{1}, \ldots, P_{h+1}\right\rangle \cap\left\langle P_{h+2}, \ldots, P_{r}\right\rangle\right\} \\
=\{ & \left(P_{1}, \ldots, P_{r}, Q\right) \in\left(\mathbb{P}^{1}\right)^{\times r} \times A \mid \operatorname{dim}\left(\left\langle Q, P_{1}, \ldots, P_{h+1}\right\rangle\right) \leq h \\
& \text { and } \left.\operatorname{dim}\left(\left\langle Q, P_{h+2}, \ldots, P_{r}\right\rangle\right) \leq r-(h+1)-1\right\} .
\end{aligned}
$$

Let $p: J \rightarrow\left(\mathbb{P}^{1}\right)^{\times r}$ and $f: J \rightarrow A$ be the natural projections. Note that $p(J) \subseteq$ $W_{r}$ because if $\left(P_{1}, \ldots, P_{r}, Q\right) \in J$ then $\left(P_{1}, \ldots, P_{r}\right)$ are not linearly independent in $\mathbb{P}^{N}$. On the other hand we have seen that for every $r$-tuple of $W_{r}$ there exists at least a point $Q \in\left\langle P_{1}, \ldots, P_{h+1}\right\rangle \cap\left\langle P_{h+2}, \ldots, P_{r}\right\rangle \subseteq A$ and that for the generic $r$-tuple of $W_{r}$ there exists a unique such $Q$. Hence $\operatorname{Im}(p)=W_{r}$ and $\operatorname{dim}(J)=$ $\operatorname{dim}\left(W_{r}\right)$. Then $\operatorname{dim}\left(W_{r}\right)=\operatorname{dim}(J)=\operatorname{dim}(\operatorname{Im}(f))+\operatorname{dim}($ generic fibre of $f) \leq$ $\operatorname{dim}(A)+\operatorname{dim}\left[f^{-1}(\bar{Q})\right]=2 h+r-N+\operatorname{dim}\left[f^{-1}(\bar{Q})\right]$ where $\bar{Q}$ is now any fixed point of $\operatorname{Im}(f)$. Pick $\bar{Q}:=\left\langle L_{1}, \ldots, L_{h+1}\right\rangle \cap L_{h+2}$. Obviously $\bar{Q} \in A$. Moreover, as $\bar{Q}$ is the intersection point of $L_{h+2}$ with $\left\langle L_{1}, \ldots, L_{h+1}\right\rangle$, we know that it does not belong to any linear subspace of $\left\langle L_{1}, \ldots, L_{h+1}\right\rangle$ spanned by a proper subset of these lines. Hence there exists a unique $(h+1)$-tuple of points $P_{1}, \ldots, P_{h+1}$, such that $\operatorname{dim}\left(\left\langle\bar{Q}, P_{1}, \ldots, P_{h+1}\right\rangle\right) \leq h$. Choosing $P_{h+2}=\bar{Q}$ one sees that there 
exists also a $(r-h-1)$-tuple of points $\left(P_{h+2}, \ldots, P_{r}\right) \in L_{h+2} \times L_{h+3} \times \cdots \times L_{r}$ such that $\operatorname{dim}\left(\left\langle\bar{Q}, P_{h+2}, \ldots, P_{r}\right\rangle\right) \leq r-(h+1)-1$. Hence $\bar{Q} \in \operatorname{Im}(f)$ and, to estimate $\operatorname{dim}\left(f^{-1}(\bar{Q})\right)$, consider the $(r-h-1)$-tuples of points $P_{h+2}, \ldots, P_{r}$ such that $\operatorname{dim}\left(\left\langle\bar{Q}, P_{h+2}, \ldots, P_{r}\right\rangle\right) \leq r-h-2$, i.e. the set $Z(\bar{Q}):=\left\{\left(P_{h+2}, \ldots, P_{r}\right) \in\right.$ $\left.L_{h+2} \times L_{h+3} \times \cdots \times L_{r} \mid \operatorname{dim}\left(\left\langle\bar{Q}, P_{h+2}, \ldots, P_{r}\right\rangle\right) \leq r-h-2\right\}$. Note that, as $r-(h+1) \geq$ $h+2 \geq 3$, we have $r \geq h+4$. Hence in $Z(\bar{Q})$ there are at least pairs of points.

Notice that, for the generic $(r-h-1)$-tuple $\left(P_{h+2}, \ldots, P_{r}\right) \in Z(\bar{Q})$, we have $\operatorname{dim}\left(\left\langle\bar{Q}, P_{h+2}, \ldots, P_{r}\right\rangle\right)=r-h-2=\operatorname{dim}\left(\left\langle P_{h+2}, \ldots, P_{r}\right\rangle\right)$. Indeed the generic $(r-h-1)$-tuple $\left(P_{h+2}, \ldots, P_{r}\right) \in Z(\bar{Q})$ is a proper subset of a generic $r$-tuple of $W_{r}$ and by Lemma 2.2, part 2), we have $\operatorname{dim}\left(\left\langle P_{h+2}, \ldots, P_{r}\right\rangle\right)=r-h-2$. On the other hand $\operatorname{dim}\left(\left\langle P_{h+2}, \ldots, P_{r}\right\rangle\right) \leq \operatorname{dim}\left(\left\langle\bar{Q}, P_{h+2}, \ldots, P_{r}\right\rangle\right) \leq r-h-2$ by the definition of $Z(\bar{Q})$. Then one can define a map $\psi: \mathcal{Z} \rightarrow W_{r-h-1}$, where $\mathcal{Z}$ is a non empty Zariski-open subset of $Z(\bar{Q})$, by setting $\psi\left(P_{h+2}, \ldots, P_{r}\right)=$ $\left(P, P_{h+3}, \ldots, P_{r}\right)$, where $\left(P_{h+2}, \ldots, P_{r}\right)$ is a generic element of $Z(\bar{Q})$ and $P$ is the unique intersection, in $\left\langle\bar{Q}, P_{h+2}, \ldots, P_{r}\right\rangle=\left\langle P_{h+2}, \ldots, P_{r}\right\rangle$ of the line $L_{h+2}$ with the linear subspace $\left\langle P_{h+3}, \ldots, P_{r}\right\rangle$. Notice that $\left\langle P_{h+3}, \ldots, P_{r}\right\rangle$ has codimension 1 in $\left\langle\bar{Q}, P_{h+2}, \ldots, P_{r}\right\rangle=\left\langle P_{h+2}, \ldots, P_{r}\right\rangle$ and it does not contain $L_{h+2}$. Obviously $\left(P, P_{h+3}, \ldots, P_{r}\right) \in W_{r-h-1}$. The generic fibre of $\psi$ is contained in $L_{h+2}$ and therefore it has dimension 1 at most. It follows that $\operatorname{dim}[Z(\bar{Q})] \leq \operatorname{dim}\left(W_{r-h-1}\right)+1$. So we get: $\operatorname{dim}[Z(\bar{Q})] \leq \operatorname{dim}\left(W_{r-h-1}\right)+1 \leq r-2 h-3+1=r-2 h-2$ by induction. Hence $\operatorname{dim}\left(W_{r}\right) \leq 2 h+r-N+\operatorname{dim}\left[f^{-1}(\bar{Q})\right] \leq 2 h+r-N+\operatorname{dim}[Z(\bar{Q})] \leq$ $2 h+r-N+r-2 h-2=2 r-N-2 \leq r-2$ and we are done.

\section{Corollaries of the Main theorem}

In this section we give a list of 5 corollaries of Theorem 2.1. The first two corollaries contain our answer to the question in Section 1. The third one proves a property of the open Zariski set $A_{r}$ which is defined in the previous corollaries. The last two show that, under the assumption $r+1 \leq N$, we can say more about the hyperplanes cutting $P_{1}, \ldots, P_{r}$ on the lines of $\mathcal{L}$.

Corollary 3.1. With the same assumptions of Theorem 2.1 there exists a non empty, Zariski-open set $A_{r} \subseteq L_{1} \times L_{2} \times \cdots \times L_{r} \simeq\left(\mathbb{P}^{1}\right)^{\times r}$ such that, for every $\left(P_{1}, \ldots, P_{r}\right) \in A_{r}$, it is $\operatorname{dim}\left(\left\langle P_{1}, \ldots, P_{r}\right\rangle\right)=r-1$, and the generic hyperplane of $\mathbb{P}^{N}$ passing through $P_{1}, \ldots, P_{r}$ does not contain any line of $\mathcal{L}$.

Proof. Let $J_{1}, J_{2}, \ldots, J_{r}$ be the $r$ varieties defined by removing, respectively, the first, the second,..., the $r^{t h}$ factor of $\left(\mathbb{P}^{1}\right)^{\times r}$. Let $p_{1}, p_{2}, \ldots, p_{r}$ be the natural projections $p_{i}:\left(\mathbb{P}^{1}\right)^{\times r} \rightarrow J_{i}$. By Theorem 2.1 we know that $\operatorname{dim}\left(W_{r}\right) \leq r-2$ in $\left(\mathbb{P}^{1}\right)^{\times r}$, hence $p_{i}^{-1}\left(p_{i}\left(W_{r}\right)\right)$ is a closed subscheme of dimension $\leq r-1$ in $\left(\mathbb{P}^{1}\right)^{\times r}$, for any $i=1, \ldots, r$. In $\left(\mathbb{P}^{1}\right)^{\times r}$, let $A_{r}$ be the complement of the union of the $r$ closed subschemes $p_{i}^{-1}\left(p_{i}\left(W_{r}\right)\right)$. Obviously $A_{r}$ is a non empty Zariski-open set in $\left(\mathbb{P}^{1}\right)^{\times r}$ and $\operatorname{dim}\left(\left\langle P_{1}, \ldots, P_{r}\right\rangle\right)=r-1$ for every $r$-tuple $\left(P_{1}, \ldots, P_{r}\right) \in A_{r}$ because $\left(P_{1}, \ldots, P_{r}\right) \notin$ $W_{r}$. Choose $L_{t} \in \mathcal{L}$ and, by contradiction, let us assume that every hyperplane in $\mathbb{P}^{N}$ passing through $P_{1}, \ldots, P_{r}$ contains $L_{t}$. This would imply that there exists a point $Q \in L_{t}\left(Q \neq P_{t}\right)$ such that $\operatorname{dim}\left(\left\langle P_{1}, \ldots, P_{t-1}, Q, P_{t+1}, \ldots, P_{r}\right\rangle\right)=$ $r-2$ and therefore $\left(P_{1}, \ldots, P_{t-1}, Q, P_{t+1}, \ldots, P_{r}\right) \in W_{r}$. In fact: if all the hyperplanes passing through $P_{1}, \ldots, P_{r}$ contain $L_{t}$, this line belongs to $\left\langle P_{1}, \ldots, P_{r}\right\rangle$, which is the intersection of all hyperplanes passing through $P_{1}, \ldots, P_{r}$; in the 
$(r-1)$-dimensional linear space $\left\langle P_{1}, \ldots, P_{r}\right\rangle$ there is the $(r-2)$-dimensional subspace $\left\langle P_{1}, \ldots, P_{t-1}, P_{t+1}, \ldots, P_{r}\right\rangle$ and the line $L_{t}$ cuts this subspace at a point $Q$. But $\left(P_{1}, \ldots, P_{t-1}, Q, P_{t+1}, \ldots, P_{r}\right)$ cannot belong to $W_{r}$ because $\left(P_{1}, \ldots, P_{r}\right) \in$ $p_{t}^{-1}\left(p_{t}\left(P_{1}, \ldots, P_{t-1}, Q, P_{t+1}, \ldots, P_{r}\right)\right)$ and if $\left(P_{1}, \ldots, P_{t-1}, Q, P_{t+1}, \ldots, P_{r}\right) \in W_{r}$ the $r$-tuple $\left(P_{1}, \ldots, P_{r}\right)$ would belong to the complement of $A_{r}$.

Corollary 3.2. With the same assumptions of Theorem 2.1, there exists a non empty, Zariski-open set $\mathcal{H} \subseteq \mathbb{P}^{N *}$ whose points correspond to hyperplanes in $\mathbb{P}^{N}$ cutting the set of lines $L_{1}, \ldots, L_{r}$ only at an $r$-tuple of points $P_{1}, \ldots, P_{r}$, with $\left(P_{1}, \ldots, P_{r}\right) \in A_{r} ;$ moreover, for any non empty Zariski-open sets $\mathcal{H}^{\prime} \subseteq \mathcal{H}$ and $A_{r}^{\prime} \subseteq A_{r}$ and for any generic $\left(P_{1}, \ldots, P_{r}\right) \in A_{r}^{\prime}$ there is at least a point in $\mathcal{H}^{\prime}$ corresponding to a hyperplane in $\mathbb{P}^{N}$ cutting the set of lines $L_{1}, \ldots, L_{r}$ only at the $r$-tuple of points $P_{1}, \ldots, P_{r}$.

Proof. To prove Corollary 3.2, let us consider the incidence variety:

$I=\left\{\left(H, P_{1}, \ldots, P_{r}\right) \in \mathbb{P}^{N *} \times\left(\mathbb{P}^{1}\right)^{\times r} \mid P_{1}, \ldots, P_{r} \in H\right\}$

and its natural projections $\alpha: I \rightarrow \mathbb{P}^{N *}$ and $\beta: I \rightarrow\left(\mathbb{P}^{1}\right)^{\times r}$. Note that $\alpha$ is surjective and the dimension of the generic fibre of $\alpha$ is zero because a generic hyperplane of $\mathbb{P}^{N}$ intersects every line of $\mathcal{L}$ at one point only; thus $\operatorname{dim}(I)=N$. For any fixed $r$-tuple of points $\left(P_{1}, \ldots, P_{r}\right) \in A_{r}$, there exists a linear subspace $\Lambda_{\left(P_{1}, \ldots, P_{r}\right)}$ in $\mathbb{P}^{N *}$, given by the hyperplanes of $\mathbb{P}^{N}$ passing through $P_{1}, \ldots, P_{r}$; we have $\operatorname{dim}\left(\Lambda_{\left(P_{1}, \ldots, P_{r}\right)}\right)=N-r$, because $P_{1}, \ldots, P_{r}$ are linearly independent. It follows that $\operatorname{dim}\left(\beta^{-1}\left(A_{r}\right)\right)=r+N-r=N$ for the non empty Zariski-open subset $\beta^{-1}\left(A_{r}\right) \subseteq I$, and therefore $I=\overline{\beta^{-1}\left(A_{r}\right)}$. Moreover, as every hyperplane either cuts every line $L_{1}, \ldots, L_{r}$ at one point only or it contains the line entirely, the generic hyperplane of $\Lambda_{\left(P_{1}, \ldots, P_{r}\right)}$ contains only the fixed $r$-tuple. If it contains other $r$-tuples it will then contain at least one of the lines in $\mathcal{L}$ but this is not possible as $\left(P_{1}, \ldots, P_{r}\right) \in A_{r}$.

The above discussion shows that a generic point of $\beta^{-1}\left(A_{r}\right)$ can be represented as a pair $\left\{H,\left(P_{1}, \ldots, P_{r}\right)\right\}$ where $H$ is a hyperplane cutting every $L_{1}, \ldots, L_{r}$ only at the points $P_{1}, \ldots, P_{r}$ with $\left(P_{1}, \ldots, P_{r}\right) \in A_{r}$. Hence there exists a subset $I^{\dagger} \subseteq$ $\beta^{-1}\left(A_{r}\right)$ given by these pairs and $I^{\dagger}$ is a non empty Zariski-open set of $I$. To see this, for any $i=1, \ldots, r$, let $C_{i}$ be the Zariski closed set in $\mathbb{P}^{N *}$ given by all hyperplanes containing $L_{i}$. Every $C_{i} \times\left(\mathbb{P}^{1}\right)^{\times r}$ is a closed set of $\mathbb{P}^{N *} \times\left(\mathbb{P}^{1}\right)^{\times r}$. Let $T$ be the complement of the union of these closed sets in $\mathbb{P}^{N *} \times\left(\mathbb{P}^{1}\right)^{\times r}$, then $I^{\dagger}$ is the intersection of the non empty Zariski-open set $T$ with $I$, so that $\overline{I^{\dagger}}=\overline{\beta^{-1}\left(A_{r}\right)}=I$. Then $\operatorname{dim}\left(\alpha\left(I^{\dagger}\right)\right)=\operatorname{dim}(\alpha(I))=N$ and therefore the interior of $\alpha\left(I^{\dagger}\right)$ is not empty. Letting $\mathcal{H}$ be the interior of $\alpha\left(I^{\dagger}\right)$, one concludes the proof of the first part of Corollary 3.2. To prove the second part it suffices to change $A_{r}$ with $A_{r}^{\prime}$ : the interior of $\alpha\left(I^{\prime \dagger}\right)$ will intersect any non empty Zariski-open set $\mathcal{H}^{\prime}$.

Corollary 3.3. With the same assumptions of Theorem 2.1, for every $L_{j} \in \mathcal{L}$ there exists a finite subset of points $K_{j} \subsetneq L_{j}$, possibly empty, such that for every point $P_{j} \in L_{j} \backslash K_{j}$, the intersection $A_{r, P_{j}}:=A_{r} \cap\left[L_{1} \times L_{2} \times \ldots,\left\{P_{j}\right\}, \cdots \times L_{r} \simeq\left(\mathbb{P}^{1}\right)^{\times(r-1)}\right]$ is an open, non empty, Zariski set of $\left(\mathbb{P}^{1}\right)^{\times(r-1)}$.

Proof. To prove Corollary 3.3 it is sufficient to remark that, as $A_{r}$ is a non empty Zariski-open set in $\left(\mathbb{P}^{1}\right)^{\times r}$, its projection onto any factor $L_{j} \simeq \mathbb{P}^{1}$ is a non empty Zariski-open set in $L_{j}$. This open set is the complement of a finite set $K_{j}$ of points (possibly empty). For every point $P_{j} \in L_{j} \backslash K_{j}, A_{r}$ can not be contained in the 
complement of the closed set $L_{1} \times L_{2} \times \ldots,\left\{P_{j}\right\}, \cdots \times L_{r}$ and $A_{r}$ intersects this closed set along a non empty Zariski-open subset of it.

Corollary 3.4. Let us assume that $r+1 \leq N$, and that there exist $r+1$ lines $L_{0}, L_{1}, \ldots, L_{r}$ satisfying the assumptions of Theorem 2.1. Let $P$ be any point on $L_{0}$ and let $\mathcal{Z}_{P} \in \mathbb{P}^{N *}$ be the dual hyperplane of $P$. Then there exists a non empty Zariski-open set $\mathcal{A}_{P} \subseteq \mathcal{Z}_{P} \simeq \mathbb{P}^{N-1}$ such that every hyperplane in $\mathbb{P}^{N}$ corresponding to a point in $\mathcal{A}_{P}$ cuts the lines $L_{1}, \ldots, L_{r}$ only at an $r$-tuple of points $P_{1}, \ldots, P_{r}$, with $\left(P_{1}, \ldots, P_{r}\right) \in A_{r}$.

Proof. Let us fix $P \in L_{0}$. By Theorem 2.1 applied to the $r+1$ lines $L_{0}, L_{1}, \ldots, L_{r}$, we have $\operatorname{dim}\left(W_{r+1}\right) \leq r-1$, hence $\operatorname{dim}\left(W_{r+1} \cap\left(\{P\} \times\left(\mathbb{P}^{1}\right)^{\times r} \simeq\left(\mathbb{P}^{1}\right)^{\times r}\right)\right) \leq$ $r-1$. Therefore there exists a non empty Zariski-open set $B_{P} \subseteq\left(\mathbb{P}^{1}\right)^{\times r}$ such that $\operatorname{dim}\left(\left\langle P, P_{1}, \ldots, P_{r}\right\rangle\right)=r$ for every choice of $\left(P_{1}, \ldots, P_{r}\right) \in B_{P}$.

By Corollary 3.1 we know that there exists a non empty Zariski-open set $A_{r}$ in $\left(\mathbb{P}^{1}\right)^{\times r}$ such that $\operatorname{dim}\left(\left\langle P_{1}, \ldots, P_{r}\right\rangle\right)=r-1$ for every choice of $\left(P_{1}, \ldots, P_{r}\right) \in A_{r}$ (and the generic hyperplane of $\mathbb{P}^{N}$ passing through $P_{1}, \ldots, P_{r}$ does not contain any line of $\mathcal{L})$. Let $C_{P}=B_{P} \cap A_{r}$. Then $C_{P}$ is a Zariski-open set in $\left(\mathbb{P}^{1}\right)^{\times r}$ such that $\operatorname{dim}\left(\left\langle P_{1}, \ldots, P_{r}\right\rangle\right)=r-1$ and $\operatorname{dim}\left(\left\langle P, P_{1}, \ldots, P_{r}\right\rangle\right)=r$ for every choice of $\left(P_{1}, \ldots, P_{r}\right) \in C_{P}$ (and the generic hyperplane of $\mathbb{P}^{N}$ passing through $P_{1}, \ldots, P_{r}$ does not contain any line of $\mathcal{L})$.

Now, to prove Corollary 3.4, let us consider the incidence variety:

$I=\left\{\left(H, P_{1}, \ldots, P_{r}\right) \in \mathcal{Z}_{P} \times L_{1} \times L_{2} \times \cdots \times L_{r} \simeq \mathbb{P}^{N-1} \times\left(\mathbb{P}^{1}\right)^{\times r} \mid P_{1}, \ldots, P_{r} \in H\right\}$ and its natural projections $\alpha: I \rightarrow \mathcal{Z}_{P}$ and $\beta: I \rightarrow\left(\mathbb{P}^{1}\right)^{\times r}$. As in the proof of Corollary 3.2, the dimension of the generic fibre of $\alpha$ is zero, because a generic hyperplane of $\mathcal{Z}_{P}$ cuts every line $L_{1}, \ldots, L_{r}$ at one point only, hence $\operatorname{dim}(I)=N-1$. For every $r$-tuple $\left(P_{1}, \ldots, P_{r}\right) \in C_{P}, \beta^{-1}\left(P_{1}, \ldots, P_{r}\right)$ is given by the hyperplanes of $\mathcal{Z}_{P}$ passing through $P_{1}, \ldots, P_{r}$, i.e. by the hyperplanes of $\mathbb{P}^{N}$ passing through $P, P_{1}, \ldots, P_{r}$. As $\operatorname{dim}\left(\left\langle P, P_{1}, \ldots, P_{r}\right\rangle\right)=r$ we have that $\operatorname{dim}\left(\beta^{-1}\left(P_{1}, \ldots, P_{r}\right)\right)=$ $N-(r+1) \geq 0$. As $C_{P}$ is a non empty Zariski-open set of $\left(\mathbb{P}^{1}\right)^{\times r}, N-(r+1)$ is also the dimension of the generic fibre of $\beta$ and therefore $\operatorname{dim}\left(\beta^{-1}\left(C_{P}\right)\right)=N-$ $(r+1)+r=N-1$ and thus $I=\overline{\beta^{-1}\left(C_{P}\right)}$. Then $\operatorname{dim}\left(\alpha\left(\beta^{-1}\left(C_{P}\right)\right)\right)=N-1$ and therefore its interior $U_{0} \subseteq \mathcal{Z}_{P}$ is not empty. Hence there exists a non empty Zariskiopen set $U_{0} \subseteq \mathcal{Z}_{P}$ such that every point of $U_{0}$ corresponds to a hyperplane in $\mathbb{P}^{N}$ containing $P$ and an $r$-tuple of points $P_{1}, \ldots, P_{r}$ with $\left(P_{1}, \ldots, P_{r}\right) \in A_{r}$. On the other hand, for every line $L_{i} \in \mathcal{L}$, there exists a non empty Zariski-open set $U_{i} \subseteq \mathcal{Z}_{P}$ given by the hyperplanes of $\mathcal{Z}_{P}$ not containing $L_{i}$. Let $\mathcal{A}_{P}=U_{0} \cap U_{1} \cap \cdots \cap U_{r}$. $\mathcal{A}_{P}$ is a non empty Zariski-open set in $\mathcal{Z}_{P}$ such that each one of its points corresponds to a hyperplane in $\mathbb{P}^{N}$ passing through $P$ and cutting the lines $L_{1}, \ldots, L_{r}$ at $r$ points $P_{1}, \ldots, P_{r}$ only, with $\left(P_{1}, \ldots, P_{r}\right) \in A_{r}$.

Corollary 3.5. With the same assumptions of Corollary 3.4, let $A_{r}^{\prime} \subseteq A_{r}$ be any non empty Zariski-open subset. Then for every point $P \in L_{0}$, there exists a non empty Zariski-open set $\mathcal{A}_{P}^{\prime} \subseteq \mathcal{Z}_{P} \simeq \mathbb{P}^{N-1}$ such that every hyperplane in $\mathbb{P}^{N}$ corresponding to a point in $\mathcal{A}_{P}^{\prime}$ cuts the lines $L_{1}, \ldots, L_{r}$ only at an $r$-tuple of points $P_{1}, \ldots, P_{r}$, with $\left(P_{1}, \ldots, P_{r}\right) \in A_{r}^{\prime}$; moreover, for any non empty Zariski-open sets $\mathcal{A}_{P}^{\prime \prime} \subseteq \mathcal{Z}_{P}$ and for any generic $\left(P_{1}, \ldots, P_{r}\right) \in A_{r}^{\prime}$ there is at least a point in $\mathcal{A}_{P}^{\prime \prime}$ corresponding to a hyperplanes in $\mathbb{P}^{N}$ cutting the set of lines $L_{1}, \ldots, L_{r}$ only at the r-tuple of points $P_{1}, \ldots, P_{r}$. 
Proof. To prove the first part of Corollary 3.5 it suffices to change $A_{r}^{\prime} \subseteq A_{r}$ with $A_{r}$ in the proof of Corollary 3.4. To prove the second part it suffices to intersect $\mathcal{A}_{P}^{\prime \prime}$ with $\mathcal{A}_{P}^{\prime}$.

\section{LINES ON RATIONAL SCROLLS}

Let $S$ be a smooth, rational, scroll surface in $\mathbb{P}^{N}$ such that $S=\mathbb{P}(\mathcal{E})$, where $\mathcal{E}=\mathcal{O}_{\mathbb{P}^{1}}(h) \oplus \mathcal{O}_{\mathbb{P}^{1}}(k)$ with $1 \leq h \leq k, N=h+k+1$, and $S$ is embedded in $\mathbb{P}^{N}$ by its tautological line bundle. Such scrolls are surfaces of minimal degree and projectively normal. By Lemma 2.1 we know that the assumptions of Theorem 2.1 are satisfied when $\mathcal{L}=\left\{L_{1}, \ldots, L_{r}\right\}$ is any set of $r$ lines in $\mathbb{P}^{N}$ which are fibres of a scroll such $S$, with $2 \leq r \leq N$. As usual $C_{0}$ and $f$ will be the numerical classes of the fundamental section and of any fibre of $S$, respectively. We have that $-C_{0}^{2}=e=k-h$, where $e$ is the invariant of $S$ (see [2, V.2] for all references about ruled surfaces).

In this section we will always assume that $\mathcal{L}=\left\{L_{1}, \ldots, L_{r}\right\}$ is a set as above and $r \geq 3$. We will show that Theorem 2.1 can be made more precise for these sets of lines when $r \geq k+2$ by using the existence of a well known incidence relation $I_{r}$, see below, however the theorem cannot be improved in this way.

First of all, let us recall that, by Lemma 2.21 ), to get any bound on the dimension on $W_{r}$, when $\mathcal{L}=\left\{L_{1}, \ldots, L_{r}\right\}$ is a set as above, we can assume that $\operatorname{dim}\left(\left\langle P_{1}, \ldots, P_{r}\right\rangle\right)=r-2$ for any generic $\left(P_{1}, \ldots, P_{r}\right) \in W_{r}$. Hence let us consider the set $\widehat{W}_{r}:=\left\{\left(P_{1}, \ldots, P_{r}\right) \in S^{(r)} \mid P_{1}, \ldots, P_{r}\right.$ are distinct, belonging to $r$ distinct lines of $S$ and $\left.\operatorname{dim}\left(\left\langle P_{1}, \ldots, P_{r}\right\rangle\right)=r-2\right\}$. Because we can choose $r$ lines amomg the fibres of $S$ in $\infty^{r}$ ways, we have $\operatorname{dim}\left(\widehat{W}_{r}\right)=\operatorname{dim}\left(W_{r}\right)+r$. Hence, to get a bound on the dimension on $W_{r}$, when $\mathcal{L}=\left\{L_{1}, \ldots, L_{r}\right\}$ is a set as above, it suffices to get a bound for the dimension of $\widehat{W}_{r}$.

Let $G$ be the Grassmannian $G(r-2, N)$ of the $(r-2)$-dimensional linear spaces of $\mathbb{P}^{N}$, let $S^{(r)}$ be the $r$-symmetric product of $S$. We can consider the incidence variety $I_{r} \subseteq S^{(r)} \times G$ such that:

$$
I_{r}:=\left\{\left(\left(P_{1}, \ldots, P_{r}\right), \Pi\right) \in S^{(r)} \times G \mid P_{1}, \ldots, P_{r} \in \Pi\right\}
$$

with the two natural projections $p: I_{r} \rightarrow S^{(r)}$ and $q: I_{r} \rightarrow G$. Note that $\widehat{W}_{r} \subseteq$ $\operatorname{Im}(p)$, moreover the fibre of $p$ over $\widehat{W}_{r}$ is given by only one $(r-2)$-dimensional linear space, so that $\operatorname{dim}\left(\widehat{W}_{r}\right)=\operatorname{dim}\left[p^{-1}\left(\widehat{W}_{r}\right)\right]$. Therefore to get bounds on the dimension of $\widehat{W}_{r}$ it is sufficient to get bounds on the dimension of $p^{-1}\left(\widehat{W}_{r}\right)$ by using $q$.

To investigate the fibre of the restriction of $q$ to $p^{-1}\left(\widehat{W}_{r}\right)$, let us put $\bar{W}_{r}:=$ $q\left[p^{-1}\left(\widehat{W}_{r}\right)\right]$ and let us consider the fibre over the generic $\Pi \in \bar{W}_{r}$. $\Pi$ is a linear space of dimension $r-2$ cutting $S$ at $r$ distinct point belonging to $r$ distinct lines of $S$. The fibre of $q$ over $\Pi$ has positive dimension, for instance, when $\Pi$ contains a curve $\Gamma$ which is a smooth, irreducible section of $S$, and in this case the fibre has dimension $r$, because we could choose any set of $r$ points on $\Gamma$. About such a section $\Gamma$, we have the following

Lemma 4.1. Let $S$ be a surface as above. Let $\Gamma$ be a smooth, irreducible section of $S, \Gamma=C_{0}+b f$, such that $\operatorname{dim}(\langle\Gamma\rangle)=N-t, t \geq 1$. Then $b=k+1-t$ and $1 \leq t \leq h+1$. 
Proof. Let $H=C_{0}+k f$ be the numerical class of the hyperplane section of $S$. Obviously $b \leq k$, and $k-h \leq b$, as $\Gamma$ is supposed to be a smooth, irreducible section of $S$. Let us consider the exact sequence: $0 \rightarrow H-\Gamma \rightarrow H \rightarrow H_{\mid \Gamma} \rightarrow 0$. As $h^{1}(S, H-\Gamma)=h^{1}\left(\mathbb{P}^{1}, \mathcal{O}_{\mathbb{P}^{1}}(k-b)\right)=0$, we have:

$N+1=h+k+2=h^{0}(S, H)=h^{0}(S, H-\Gamma)+h^{0}\left(\Gamma, H_{\mid \Gamma}\right)=(k-b+1)+(N-t+1)$.

Hence: $b=k+1-t$ and it must be: $k-h \leq k+1-t \leq k$, i.e. $1 \leq t \leq h+1$.

Now let us return to the fibre over the generic $\Pi \in \bar{W}_{r}$. By Lemma 4.1, if $\Pi$ contains a section $\Gamma$ as above, given that $\langle\Gamma\rangle \subseteq \Pi$, then $N-t \leq r-2$ with $t \leq h+1$, hence $N+2-r \leq h+1$, hence $h+k+3-r \leq h+1$, hence $r \geq k+2$. It follows that $r \geq k+2$ is exactly the range for which sections as $\Gamma$ can occur.

Let $V$ be the subvariety of $G$ parametrizing $(r-2)$-dimensional linear spaces of $\mathbb{P}^{N}$ which are $(r-1)$-secant $S$. Obviously $\operatorname{Im}(q) \subseteq V$, but $\operatorname{Im}(q) \neq V$ and $\operatorname{dim}(V)=$ $2(r-1)$, so that $\operatorname{dim}(\operatorname{Im}(q))<2 r-2$. If $\operatorname{dim}\left\{q\left[p^{-1}\left(\widehat{W}_{r}\right)\right]\right\}=\operatorname{dim}\left[p^{-1}\left(\widehat{W}_{r}\right)\right]$, then $\operatorname{dim}\left(\widehat{W}_{r}\right)=\operatorname{dim}\left[p^{-1}\left(\widehat{W}_{r}\right)\right]<2 r-2$, hence $\operatorname{dim}\left(W_{r}\right)<r-2$ thus giving a stronger bound; but we saw above that fibres of the restriction of $q$ to $p^{-1}\left(\widehat{W}_{r}\right)$ can be of positive dimension when $r \geq k+2$, hence we cannot use $I_{r}$ to improve Theorem 2.1. However we can prove the following

Proposition 4.1. Let $S$ be a surface as above and let $\widehat{W}_{r}$ be defined as above. Assume that $N \geq r \geq k+2$, then $\operatorname{dim}\left(\widehat{W}_{r}\right)=2 r-2$ and $\operatorname{dim}\left(W_{r}\right)=r-2$.

Proof. We know that $\operatorname{dim}\left(\widehat{W}_{r}\right)=\operatorname{dim}\left(W_{r}\right)+r$, so we can consider only $\widehat{W}_{r}$. By Theorem 2.1 it is sufficient to show that $\operatorname{dim}\left(\widehat{W}_{r}\right) \geq 2 r-2$.

Let us put $r=k+2+\eta$ with $0 \leq \eta \leq h-1$. Utilizing again the incidence relation $(*)$ introduced above, we will show that $\operatorname{dim}\left(\widehat{W}_{r}\right)=\operatorname{dim}\left[p^{-1}\left(\widehat{W}_{r}\right)\right] \geq e+2 \eta+1+r$ for any $\eta$ with $0 \leq \eta \leq h-1$. By choosing $\eta=h-1$ we will have $\operatorname{dim}\left[p^{-1}\left(\widehat{W}_{r}\right)\right] \geq$ $2 r-2$.

Let us fix $k-(e+\eta)=h-\eta \geq 1$ distinct fibres on $S$ and let us consider all hyperplanes in $\mathbb{P}^{N}$ containing such fibres: $h^{0}(S, H-(h-\eta) f)=h^{0}\left(S, C_{0}+(e+\right.$ $\eta) f)=e+2 \eta+2 \geq 2$. This means that on $S$ there exist a family of dimension at least $e+2 \eta+1$ of curves $\Gamma=C_{0}+(e+\eta) f$, possibly reducible, such that $\operatorname{dim}(\langle\Gamma\rangle)=N-h^{0}(S, H-\Gamma)=N-h^{0}\left(\mathbb{P}^{1}, \mathcal{O}_{\mathbb{P}^{1}}(h-\eta)\right)=N-(h-\eta+1)=r-2$. Note that in any $\langle\Gamma\rangle \simeq \mathbb{P}^{r-2}$ there are at most a finite number of curves as $\Gamma$, otherwise $S$ would be contained in a projective space of dimension $r-2<N$.

Now let us recall the incidence variety $I_{r}$ : by the previous remark we have that the subvariety $V_{\Gamma} \subseteq \bar{W}_{r} \subseteq G$ parametrizing subspaces as $\langle\Gamma\rangle$ has dimension at least $e+2 \eta+1$, hence $\operatorname{dim}\left(\bar{W}_{r}\right) \geq e+2 \eta+1$, moreover the fibre of $q$ over any point of $V_{\Gamma}$ has dimension at least $r$, hence $\operatorname{dim}\left[p^{-1}\left(\widehat{W}_{r}\right)\right] \geq e+2 \eta+1+r$.

\section{A Simple APPLiCATiON}

To conclude the paper we give a simple application of Corollary 3.2. As mentioned in the introduction, this was the original situation that brought us to consider the problem addressed in this note.

Proposition 5.1. Let $\left\{S_{1}, S_{2}\right\}$ be a pair of surfaces in $\mathbb{P}^{N}$ as in Section 4. Assume that the intersection $S_{1} \cap S_{2}$ in $\mathbb{P}^{N}$ consists only of $r$ common fibres $L_{1}, \ldots, L_{r}$ and that, at a generic point $P \in L_{i}$, the tangent planes to $S_{1}$ and $S_{2}$ at $P$ are distinct. 
Then, for any generic choice of $r$ points $P_{1}, \ldots, P_{r}, P_{i} \in L_{i}$, there is a hyperplane of $\mathbb{P}^{N}$ intersecting transversally $S_{1} \cap S_{2}$ only at $P_{1}, \ldots, P_{r}$.

Proof. Apply Corollary 3.2 to $\mathcal{L}:=\left\{L_{1}, \ldots, L_{r}\right\}$, keeping in mind that the assumptions of Theorem 2.1 are satisfied for any set of $r$ fibres on surfaces as above.

Remark 5.1. Note that the set up of Proposition 5.1 is achieved, for instance, when every $S_{j}$ is $\mathbb{P}\left(\mathcal{E}_{\mid \Gamma_{j}}\right)$, where $\mathcal{E}$ is a rank 2 vector bundle over a smooth variety $Y, \Gamma_{1}$ and $\Gamma_{2}$ are rational curves in $Y$ whose intersection is transverse and consists of $r$ distinct points, and $\mathbb{P}(\mathcal{E})$ is embedded in $\mathbb{P}^{N}$ as a scroll.

Following Remark 5.1, let $\mathcal{E}$ be a rank 2 vector bundle over a smooth surface $Y$ which is rationally connected; let $X$ be $\mathbb{P}(\mathcal{E})$, let $T$ be its tautological divisor and let $\pi: X \rightarrow Y$ be the natural projection. In order to prove that the linear system $|T|$ separates two distinct points $P$ and $Q$ of $X$ you can consider a rational smooth curve $\Gamma$ (if it exists) passing through $\pi(P)$ and $\pi(Q)$, and the surface $S:=\mathbb{P}\left(\mathcal{E}_{\mid \Gamma}\right)$. If $|T|_{\mid S}$ is very ample and $|T| \rightarrow|T|_{\mid S}$ is surjective then $|T|$ separates $P$ from $Q$. The difficult part of this strategy is often to prove the surjectivity (see for instance $[1])$. The usual exact sequence $0 \rightarrow \mathcal{E} \otimes \mathcal{O}_{Y}(-\Gamma) \rightarrow \mathcal{E} \rightarrow \mathcal{E}_{\mid \Gamma} \rightarrow 0$ gives the required surjectivity if $h^{1}\left(Y, \mathcal{E} \otimes \mathcal{O}_{Y}(-\Gamma)\right)=0$. Unfortunately, this vanishing is not always easy to control. One may choose a set $\left\{\Gamma=\Gamma_{1}, \ldots \Gamma_{q}\right\}$ of $q>>1$ suitable smooth rational curves in order to get $h^{1}\left(Y, \mathcal{E} \otimes \mathcal{O}_{Y}\left(-\Gamma_{1} \ldots-\Gamma_{q}\right)\right)=0$ and then use a reducible surface $S^{\prime}:=S_{1} \cup \ldots \cup S_{q}$, instead of $S$, with $S_{j}:=\mathbb{P}\left(\mathcal{E}_{\mid \Gamma j}\right)$. With this approach one needs to consider elements of $|T|_{S^{\prime}}$. Even when $|T|_{\mid S_{j}}$ is very ample for any $j$, and $\Gamma_{i} \cap \Gamma_{j}$ is a set of distinct points for any $i, j$, to get sections of $|T|_{S^{\prime}}$ it is crucial to know what elements of $|T|$ cut $S_{i} \cap S_{j}$ only at distinct points. Proposition 5.1 gives the answer.

\section{REFERENCES}

[1] A. Alzati - G.M. Besana: "Criteria for very ampleness of rank two vector bundles over ruled surfaces" Can J. Math. 62 (6) pp 1201-1227 (2010).

[2] R. Hartshorne: "Algebraic Geometry" Springer-Verlag, New York, 1977.

Dipartimento di Matematica Univ. di Milano, via C. Saldini 50 20133-Milano (Italy)

E-mail address: alberto.alzati@unimi.it

College of Computing and Digital Media, DePaul University, 1 E. Jackson Blvd ChicAgo IL 60604 USA

E-mail address: gbesana@depaul.edu 